\title{
Target site analysis of RTE1_LA and its AfroSINE partner in the elephant genome
}

\author{
Clément Gilbert $^{\mathrm{a}, \mathrm{b}, *}$, John K. Pace $\mathrm{II}^{\mathrm{b}}$, Paul D. Waters ${ }^{\mathrm{a}, \mathrm{c}}$ \\ a Evolutionary Genomics Group, Department of Botany and Zoology, University of Stellenbosch, Stellenbosch, South Africa \\ b Department of Biology, University of Texas, Arlington, Texas 76019, USA \\ c Comparative Genomics Group, Research School of Biological Sciences, The Australian National University, Canberra, ACT, Australia
}

\section{A R T I C L E I N F O}

Article history:

Received 14 May 2008

Received in revised form 18 August 2008

Accepted 18 August 2008

Available online 28 August 2008

Received by M. Batzer

\begin{abstract}
A B S T R A C T
SINEs retrotranspose using their partner LINE's enzymatic machinery. It has recently been proposed that AfroSINEs ending with GGTTT 3' tandem repeats were mobilized by RTE elements ending with CAA 3' tandem repeats in the Afrotherian genome. Using sequences from the elephant genome, we show that AfroSINEs derive from RTE ending with GGTTT-like 3' tandem repeats, a subgroup of RTE1_LA that only reached low copy number, and confirm that they were most likely mobilized by RTE ending with $\mathrm{CAA}_{(\mathrm{n})}$ tandem repeats $\left(\right.$ RTE1_LA-CAA $\left.A_{(n)}\right)$. This partnership is supported by sequence similarity between two regions of the elements, overlap in the timing of their activity, common features of their target site consensus that are not shared by other members of the RTE family, and their high copy number. Detailed analyses of pre-insertion loci reveal that like many other apurinic/apyrimidinic endonuclease encoding elements, RTE1_LA-CAA $(\mathrm{n})$ shows loose target site specificity. In addition, the RTE1_LA-CAA $(\mathrm{n})$ target site consensus shares several structural and primary sequence features with that of LINE1, suggesting that these two elements share close functional similarity in the target primed reverse transcription (TPRT) reaction. Interestingly, although globally similar, the target site consensus of AfroSINE(Anc) and RTE1_LA-CAA $(\mathrm{n})$ differ in several aspects. These differences, not observed among all SINE/LINE pairs so far examined, are most likely due to the fact that AfroSINEs and RTE1_LA-CAA $(\mathrm{n})$ are terminated by a different tandem repeat motif. We propose that these differences reflect constraints imposed by base pairing interactions between the mRNA 3' terminal tandem repeats and the target DNA at the onset of TPRT. So in addition to the endonuclease nicking preference, the mRNA of these elements appears to play an important role in integration site choice through a passive, post-nicking, selective process.
\end{abstract}

(C) 2008 Elsevier B.V. All rights reserved.

\section{Introduction}

Non-long terminal repeat retrotransposons (NLR) have been the most successful transposable elements to colonize mammalian genomes (e.g., Lander et al., 2001; Gentles et al., 2007). Canonical replication of these elements occurs via target site primed reverse transcription (TPRT) of their mRNA. The target DNA is cleaved on the bottom DNA strand by the element encoded endonuclease (EN), producing a free $3^{\prime}-\mathrm{OH}$ group that is utilized by the element encoded reverse transcriptase (RT) in the presence of the element mRNA to prime reverse transcription (Luan et al., 1993; Cost et al., 2002). It has been shown that base pairing between the target DNA and the 3' end of the mRNA might be required for, or at least facilitate the initiation of the TPRT for the I factor and the R1Bm elements (Chaboissier et al., 2000;

Abbreviations: AP, apurinic/apyrimidinic; EN, endonuclease; LINE, long interspersed nuclear element; NLR, non-long terminal repeat retrotransposon; ORF, open reading frame; RT, reverse transcriptase; SINE, short interspersed nuclear element; TPRT, target primed reverse transcription; TSD, target site duplication.

* Corresponding author.

E-mail address: clement.gilbert@uta.edu (C. Gilbert).
Anzai et al., 2005). The extent to which these interactions are ubiquitous among NLRs is not clear. However, it seems clear that for some elements (like R2Bm) these interactions are not required for TPRT (Luan and Eickbush, 1995). After completion of the reverse transcription, second strand synthesis of the element is performed through a process that remains to be fully described (Kurzynska-Kokorniak et al., 2007). TPRT generally results in the duplication of a short stretch of nucleotides (generally $<20 \mathrm{bp}$ ) located between the two cleavage sites. Thus, each newly inserted element is typically flanked by short direct repeats, also called target site duplication (TSD).

NLRs can be divided into two groups (reviewed in Kazazian, 2004). LINEs (long interspersed nuclear element) are between 3 and $10 \mathrm{~kb}$ and considered to be autonomous because they encode most if not all the factors required for the TPRT. By contrast, non-autonomous elements (SINEs, SVA [named after SINE,VNTR (variable number of tandem repeats) and $A l u$ ] and processed pseudogenes) are generally much shorter (90 to $400 \mathrm{bp}$ for SINEs) and cannot rely on self-encoded enzymes for the TPRT since they do not possess open reading frames (ORFs). Most SINEs have a chimeric structure which, in most instances, consists of a 5' tRNA-related region that contains the A and B boxes of the PolIII promoter, followed by a core region of unknown origin, and a 
$3^{\prime}$ region that shares high similarity to the $3^{\prime}$ region of a LINE family present in the same host genome (reviewed in Ohshima and Okada, 2005). Luan et al. (1993) showed that this $3^{\prime}$ region is pivotal in the initiation of the TPRT of the R2 LINE element of Bombyx mori, as it is specifically recognized by the 3' RNA binding domain of this element. This, and other evidence, led to the hypothesis that SINEs could parasitize the enzymatic machinery encoded by LINEs through this shared 3' region (Smit and Riggs, 1995; Ohshima et al., 1996; Jurka, 1997), which was experimentally validated for several SINE/LINE pairs (Kajikawa and Okada, 2002; Dewannieux et al., 2003; Dewannieux and Heidmann, 2005; Kajikawa et al., 2005).

Many NLRs show a preference for insertion at specific target sites (reviewed in Zingler et al., 2005). Target site specificity as well as its determinants vary broadly among NLRs. Although some exceptions exist (Volff et al., 2001; Mandal et al., 2004), NLRs encoding a restriction enzyme-like endonuclease usually have a strong target site preference, inserting exclusively into a specific site within a particular type of sequence such as, for example, rRNA genes, simple repeats or mini-exon arrays (Burke et al., 1987; Aksoy et al., 1990; Gabriel et al., 1990; Burke et al., 1995; Burke et al., 2003). The specificity of these elements is determined by their DNA binding domains (e.g., Christensen et al., 2005). By contrast, NLRs encoding an apurinic/ apyrimidinic (AP) endonuclease generally show a weak target site consensus and can insert at many different loci within their host genome (Szak et al., 2002; Bringaud et al., 2006; reviewed in Zingler et al., 2005), although some notable exceptions exist (Garrett et al., 1989; Higashiyama et al., 1997; Feng et al., 1998; Kojima and Fujiwara, 2003). For both the specific and non-specific AP endonuclease encoding elements, the AP endonuclease is the primary determinant for recognition and nicking of the target DNA (Cost and Boeke, 1998; Feng et al., 1998). However, integration at a specific site also depends on other factors, such as the structural parameters of the target DNA (Cost and Boeke, 1998; Zingler et al., 2005; Repanas et al., 2007).

It was recently proposed that AfroSINEs derive from, and were mobilized by, RTE LINEs in the Afrotherian genome because these two elements share two regions of high sequence similarity (Gogolevsky et al., 2008). Intriguingly, contrary to the majority of SINE/LINE pairs so far described in eukaryotes, AfroSINEs and known Afrotherian RTEs are not terminated by the same tandem repeat motifs. In this study, we first explain this peculiarity of the AfroSINE/RTE pair and refine the origin of AfroSINEs by taking advantage of sequences from the elephant genome. We then conduct analyses of sequence conservation at the pre-insertion loci of elephant AfroSINEs and RTEs, and discuss the outcomes in light of our current knowledge on the TPRT reaction and the partnership between LINEs and SINEs. Our results support the view that the element mRNA plays a critical role in integration dynamics, most likely through passive integration site selection involving interactions between its $3^{\prime}$ end and the target DNA.

\section{Materials and methods}

\subsection{Repeat masking}

Estimates of copy number of AfroSINEs and RTE elements in the current version of the elephant genome (May 2005) were performed using a local version of RepeatMasker and custom libraries that included either the AfroSINE(Anc) (Nikaido et al., 2005) or the RTE1_LA (Jurka, 2006) consensus. Search for RTE elements ending with GGTTTlike terminal tandem repeats was conducted using the RTE1_LA consensus in which CAA terminal repeats were replaced with GGTTT repeats. In order to circumvent the problem of cross match between AfroSINEs and RTEs (due to the high similarity between two regions of the elements), we considered only elements that had a $3^{\prime}$ region longer than 200 bp for RTEs and longer than 150 bp for AfroSINEs. Average similarity between each copy and the consensus was derived from the RepeatMasker output.

\subsection{Analysis of sequence conservation at pre-insertion loci}

Blastn searches were performed on the draft elephant genome in Genbank using the consensus of the two partner elements as query sequences. 1000 AfroSINE(Anc)- and 1000 RTE1_LA-containing contigs were downloaded. Full-length elements with perfect target site duplication (TSD) or with a maximum of one mismatch between the $5^{\prime}$ and $3^{\prime}$ copy of the TSD were retained for analysis. Both ends of the TSD were manually inspected using BioEdit v5.9 (Hall, 2004). Only TSDs meeting the following criteria were retained for analysis: the two base pairs immediately flanking both ends of the TSD must be different between the $5^{\prime}$ and the $3^{\prime}$ copy of the TSD, there must be at least one mismatch within the two following flanking bp, and no obvious similarity thereafter. TSDs as well as $200 \mathrm{bp}$ of $5^{\prime}$ and $3^{\prime}$ flanking regions were aligned using BioEdit v5.9 (Hall, 2004) as presented in Jurka (1997); no attempt was made to maximize homology but sites were organized according to their position with regard to the start and the end of the elements. When present, mismatched positions between the $5^{\prime}$ and $3^{\prime}$ copy of the TSD were treated as gaps. Analyses of sequence conservation follow Schneider and Stephens (1990) and were performed using the Web Logo server (http://weblogo.berkeley. edu; Crooks et al., 2004).

\section{Results and discussion}

3.1. Origin of AfroSINEs and implications on their partnership with RTE elements

The 3' region of AfroSINEs shares similarity (81\% over $55 \mathrm{bp}$ ) with the 3 ' region of the RTE1_LA element characterized in the elephant genome (Jurka, 2006). In addition, their central region is also similar (74.5\% over 57 bp) to a fragment of the $5^{\prime}$ region of RTE1_LA. Based on these observations, Gogolevsky et al. (2008) suggested that AfroSINEs derive from RTE elements and were mobilized by the RTE encoded enzymatic machinery. However, although presumed partners, AfroSINEs and RTE1_LA do not end with the same 3' tandem repeat motif (situated 3' of the region shared by the two elements), i.e., respectively $\operatorname{GGTTT}_{(\mathrm{n})}$ and $\mathrm{CAA}_{(\mathrm{n})}$. This difference could be explained in two ways: (1) AfroSINEs derive from an RTE ending with CAA repeats that were replaced by the GGTTT motif, possibly through template slippage during the early stages of the TPRT reaction (Luan and Eickbush, 1995; Chaboissier et al, 2000; Tu et al., 2004) and/or via mutations introduced during reverse transcription or after insertion, followed by expansion/contraction through replication slippage and/or recombination (Arcot et al., 1995); (2) AfroSINEs derive from hitherto uncharacterized RTE elements ending with a GGTTT-like tandem repeat motif. A search of the elephant genome using RepeatMasker and the consensus RTE1_LA where CAA 3' repeats were replaced with GGTTT repeats yielded $4233^{\prime}$ regions of RTE elements longer than $200 \mathrm{bp}$ that ended with GGTTT-like tandem repeats. According to this result, it is more parsimonious to assume that AfroSINEs derive from RTE with GGTTTlike repeats rather than from RTE ending with CAA repeats (Fig. 1).

An interesting question that arose from the discovery of RTE elements ending with GGTTT-like repeats is which type of RTE mobilized AfroSINEs. To answer this, the relationships between the three elements, as well as their amplification dynamics must be clarified. RTE elements ending with GGTTT-like repeats only reached a very low copy number in the elephant genome. Of 423 elements, only three are full-length and 345 are less than $1 \mathrm{~kb}$. To derive a consensus for these elements, all fragments longer than $1 \mathrm{~kb}$ were aligned. Similarity between this consensus and that of RTE ending with CAA 3' repeats is $95 \%$.

We conducted phylogenetic analyses of a $1 \mathrm{~kb}$ alignment that includes the 78 RTE ending with GGTTT-like repeats of at least $1 \mathrm{~kb}, 78$ randomly selected RTE1_LA, and the RTE element of opposum, ruminant and vipera as outgroups. The results show that all copies of 

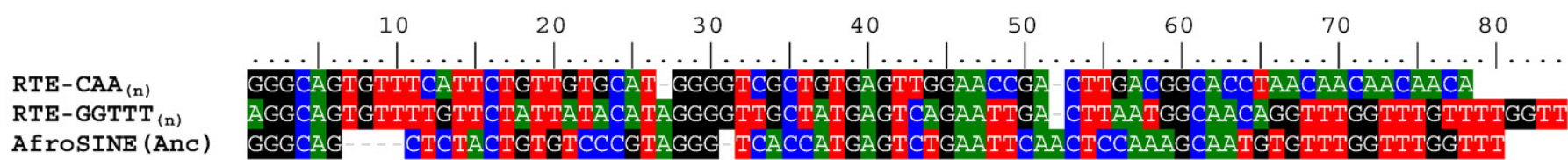

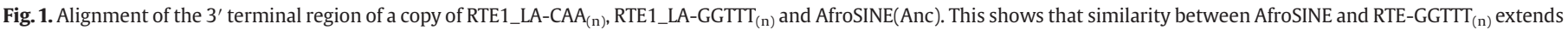

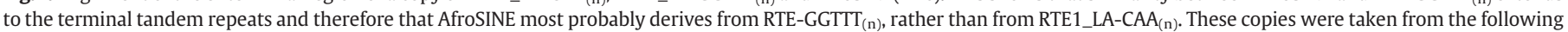

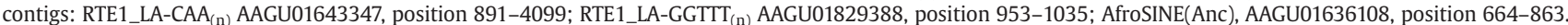

RTE1_LA and RTE ending with GGTTT-like repeats form a strongly supported clade (bootstrap=100), but that within this clade, RTEs ending with GGTTT-like repeats do not group together as a monophyletic subfamily (see Supplementary Fig. S1). Therefore, RTEs ending with GGTTT-like repeats are not a different family distantly related to RTE1_LA, rather they constitute a subset of RTE1_LA. We will use the terms RTE1_LA-GGTTT $(\mathrm{n})$ and RTE1_LA-CAA $(\mathrm{n})$ to refer to these two different subsets for the rest of the manuscript. Copy number of AfroSINEs and RTE1_LA-CAA $(\mathrm{n})$ was estimated in the draft elephant genome using RepeatMasker. We found 118,953 3' regions of RTE1_LA$\mathrm{CAA}_{(\mathrm{n})}$ longer than $200 \mathrm{bp}$ and 313,448 $3^{\prime}$ regions of AfroSINEs longer than $150 \mathrm{bp}$. It therefore appears that these two elements were much more successful than RTE1_LA-GGTTT $(\mathrm{n})$, reaching copy numbers in same order of magnitude as the RTE and associated SINEs in the opposum genome (Gentles et al., 2007).

Average similarity of all copies to their full-length consensus is 85.7\% (SD = 5.5\%) for RTE1_LA-CAA ${ }_{(\mathrm{n})}, 84.2 \%(\mathrm{SD}=5.7 \%)$ for RTE1_LAGGTTT $_{(\mathrm{n})}$ and $82.3 \%(\mathrm{SD}=5.1 \%)$ for AfroSINE. $\mathrm{G}+\mathrm{C}$ content for each consensus is 42\% $\left(\right.$ RTE1_LA-CAA $\left._{(\mathrm{n})}\right)$, 41\% (RTE1_LA-GGTTT $(\mathrm{n})$ ) and $50.6 \%$ (AfroSINE). Average similarity to the 3 ' shared region that has an almost identical nucleotide composition between the three elements is $84.5 \%(\mathrm{SD}=4.8 \%)$ for RTE1_LA-CAA $(\mathrm{n})(60 \mathrm{bp}$; $51.7 \% \mathrm{G}+\mathrm{C}), 82.9 \%$ $(\mathrm{SD}=4.6 \%)$ for RTE1_LA-GGTTT $(\mathrm{n})(60 \mathrm{bp} ; 51.7 \% \mathrm{G}+\mathrm{C})$ and $83.4 \%$ $(\mathrm{SD}=4.8 \%$ ) for AfroSINE (56 bp; $50 \% \mathrm{G}+\mathrm{C}$ ).

Overall, these data indicate that the three elements are of similar age and that, therefore, their dates of activity overlapped. This implies that AfroSINEs could have potentially been mobilized by either, or both, RTE1_LA subgroups. However, it is clear that given the large difference in copy numbers between the two RTE1_LA subgroups, the activity level of RTE1_LA-CAA $(\mathrm{n})$ has been by far higher than that of RTE1_LA-GGTTT $(\mathrm{n})$, implying that RTE1_LA-CAA $(\mathrm{n})$ has most probably been the main if not the only source of RTE encoded enzymes during the history of the elephant genome. Moreover, although examples where only few copies of autonomous elements gave rise to thousands of non-autonomous elements are common for DNA transposons (Feschotte et al., 2002, 2003), this has not yet been observed for NLRs, probably because of the cis-preference (the preferential reverse transcription of its own mRNA by the RT) shown by these elements during transposition (Esnault et al., 2000; Wei et al., 2001; Kajikawa and Okada, 2002). Therefore, although AfroSINEs most probably derive from RTE1_LA-GGTTT $(\mathrm{n})$, we believe that they have been mainly if not only mobilized by RTE1_LA-CAA $(\mathrm{n})$.

\subsection{Target site analysis of AfroSINE(Anc) and RTE1_LA-CAA $(n)$ in the elephant genome}

Along with the fact that RTE1_LA-CAA $(\mathrm{n})$ and AfroSINE share two regions of high similarity, overlap in their timing of activity and high copy numbers suggest that they are partners. However, we acknowledge that a definitive conclusion on the ability of AfroSINE to parasitize RTE1_LA-CAA $(\mathrm{n})$ would require experimental data. Nonetheless, in order to further test this presumed partnership, we conducted analyses of sequence conservation at the pre-insertion loci of these two elements in the elephant genome.

\subsubsection{Loose specificity of the RTE1_LA-CAA $A_{(n)}$ encoded endonuclease}

Enough perfect or nearly perfect target sites to perform statistical analyses were found only for RTE1_LA-CAA $(\mathrm{n})$ and AfroSINE of the "Ancestral" subfamily (Anc) (Nikaido et al., 2005). From 2000 elephant contigs, 85 full-length RTE1_LA-CAA ${ }_{(\mathrm{n})}$ and 99 full-length AfroSINE (Anc) flanked by perfect, or nearly perfect TSDs were identified (see Materials and methods). A+T content of the TSD was high for both RTE1_LA-CAA $(\mathrm{n})$ and AfroSINE(Anc) (67\% and 68\% respectively). TSD length varied from 7 to $13 \mathrm{bp}$ for RTE1_LA-CAA $(\mathrm{n})($ mode $=11 \mathrm{bp}$ ) and from 7 to $16 \mathrm{bp}$ for AfroSINE(Anc) (mode $=12 \mathrm{bp}$ ) (Fig. 2). A+T rich TSDs are also generally observed for RTE elements described in other taxa (Malik and Eickbush, 1998; Laha et al., 2002, 2005; Gentles et al., 2007; Ichiyanagi and Okada, 2008). Furthermore, based on the complete length profile of RTE TSDs in cow, opossum and zebrafish, Ichiyanagi and Okada (2008) reported a most frequent TSD length of 10-12 bp, similar to what is observed here. The similar TSD length and A+T content observed between AfroSINE(Anc) and RTE1_LA-CAA $(\mathrm{n})$ is consistent with their TPRT being mediated by the same enzymes.

The 99 AfroSINE(Anc) and 85 RTE1_LA-CAA $(n)$ 's TSDs aligned from the first $5^{\prime}$ base are presented in Fig. 3 and Supplementary Fig. S2, respectively. Because of the heterogeneous length of the TSDs, two
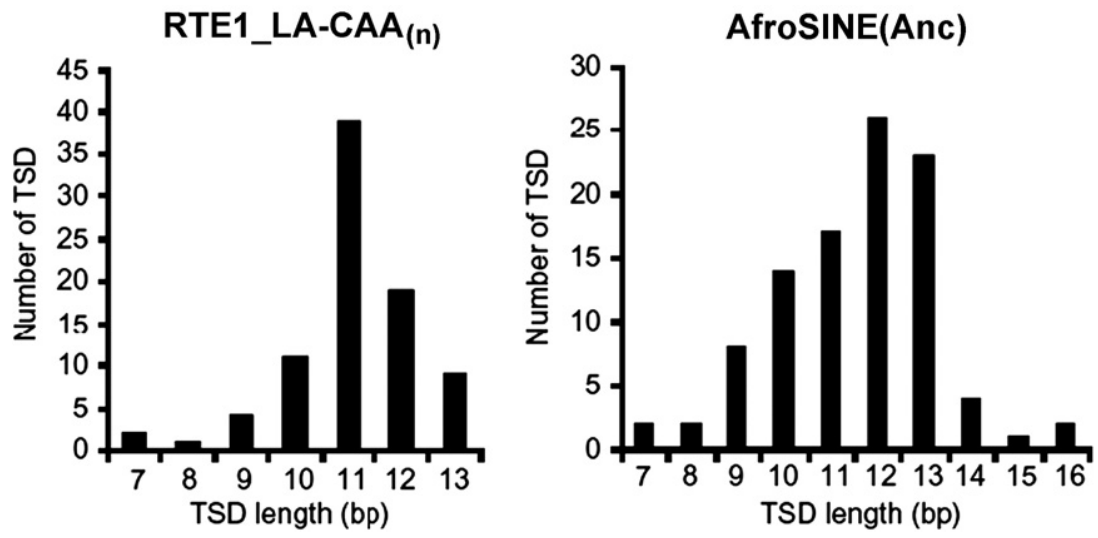

Fig. 2. Distribution of the TSD lengths for RTE1_LA-CAA $(n)(n=85)$ and AfroSINE(Anc) $(n=99)$. 


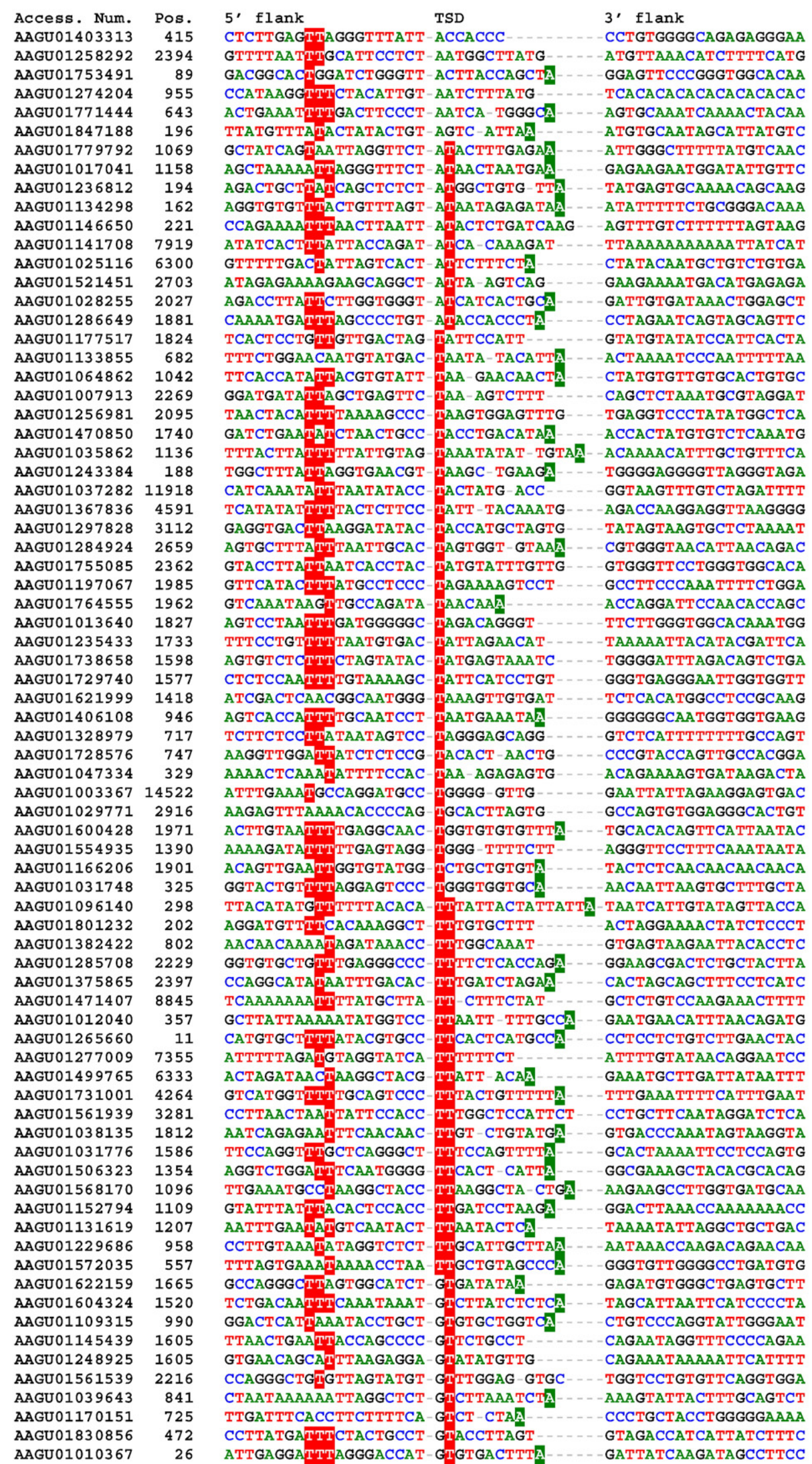

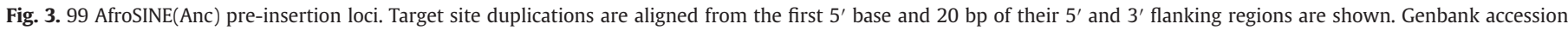

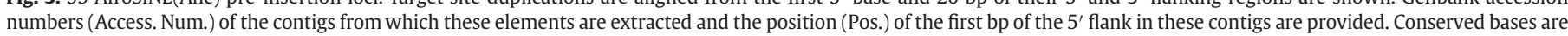
boxed (see Logo graphs, Fig. 4). Gaps within TSDs correspond to mismatches between the two TSD copies. 


\begin{tabular}{|c|c|c|}
\hline Access. Num. & & $3^{\prime}$ flank \\
\hline AAGU01589129 & 172 & СTGGGTTTGTCATTGGATTT \\
\hline AAGU01125568 & 303 & ---ACTTTTCTGAGCATTGAGAA \\
\hline AAGU01096147 & 1499 & --CTGCCTAGGGTGAGGCTGGT \\
\hline AAGU01157200 & 6937 & - CGGATTCCATTCTACCAGCA \\
\hline AAGU01030522 & 3707 & -ATTGGTGTGTTTATTGTATG \\
\hline AAGU01206894 & 1924 & --TCСАTTTTTCСTTATGTCCA \\
\hline AAGU01691573 & 394 & GGATGTTAATAGCATCATT-GCTTC-AAGT" \\
\hline AAGU01581333 & 484 & - CAGATGGGAAAACGGAGGAC \\
\hline AAGU01251604 & 869 & -- ATGGAAATGACTTCAGCACA \\
\hline AAGU01837872 & 123 & - - GCAACATATGTACTTAAGAG \\
\hline AAGU01010444 & 1321 & - - GCAAATTGCCAGATCTTTCT \\
\hline AAGU01031530 & 1370 & - AGCTGAATGAAACAAACTAC \\
\hline AAGU01060450 & 1381 & AGGAGAAACATGCACTGAT-GATCAG-AATC \\
\hline AAGU01150579 & 2718 & CAAA-GCAGCCGTGGCGGCAGAATG \\
\hline AAGU01218881 & 3776 & - GAAAAAAACTACCACAGATA \\
\hline AAGU01163875 & 781 & - AGGAGATTGCCAGGCTTTTC \\
\hline AAGU01610627 & 49 & ---GGTGCAGCTGAAAGGCTACT \\
\hline AAGU01148763 & 593 & - CAGATGAGTAAACTAAGGCA \\
\hline AAGU01030759 & 1317 & - GCATTGATTGTGGATCCAAG \\
\hline AAGU01039850 & 1764 & AGGTAGTATAATGACCTGGC-AATATGTTCA \\
\hline AAGU01015795 & 3816 & - TCCTCATCTGTGGAATGGAG \\
\hline AAGU01135410 & 5780 & AATAAATTTTGAGAACCACT-GCTTT-CTAGT-_-GGTTCTCAGAATGGGTCCCT \\
\hline AAGU01146008 & 1 & - СTAATGAATTTTAATAGAC-ACATGC-AGTGAA- - -AATATCACTGCACACGGTAT \\
\hline
\end{tabular}

Fig. 3 (continued).

analyses of sequence conservation were performed following Jurka (1997); one with the TSDs aligned from the first 5' base, and one with the TSDs aligned from the first $3^{\prime}$ base (see Figs. 3 and 4). Logo graphs resulting from the two analyses are presented on Fig. 4a-d. As there was no overlap of conserved sites between the two alignments, Logo graphs were combined for each element (Fig. 4e and f) to illustrate more clearly the pattern of sequence conservation flanking the two nicking sites. This shows that both elements have relative target site specificity, with a loose top strand consensus of $5^{\prime}-\operatorname{TTTN}_{(9)} \mathrm{T}\left|\mathrm{AN}_{(10)}\right|$ A - for RTE1_LA-CAA $(n)$, and 5'-TTTN $(9)\left|\operatorname{TTN}_{(9)} \mathrm{A}\right|-$ for AfroSINE (Anc); where "|" represents the nicking sites. Similarly loose target site consensuses were recently reported by Ichiyanagi and Okada, (2008) for the RTE elements of cow, opossum and zebrafish.

All RTE described so far encode an N-terminal apurinic/apyrimidinic endonuclease (APE) domain. Comparison with the well-characterized RTE element of the viper (Zupunski et al., 2001) reveals that this domain is also present in RTE1_LA (position 396-1127 of the repbase consensus; not shown). A generally weak (or even no) specific target site preference has so far been reported for APE-type NLRs (reviewed in Zingler et al., 2005), which is consistent with the loose target site consensus observed here for RTE1_LA-CAA $(\mathrm{n})$ and AfroSINE(Anc).

\subsubsection{Comparison with the $L 1$ target site consensus}

To our knowledge, the only other APE-type NLRs for which the pattern of sequence conservation is well described around both nicking sites is the L1 element and its associated SINEs (Jurka and Klonowski, 1996; Jurka, 1997; Szak et al., 2002; Gentles et al., 2005). The first nick (bottom strand) of L1 AP endonuclease preferentially occurs at the $5^{\prime}-\mathrm{TT} \mid$ AAAA-3' consensus, and the second nick (top strand) at the 5'-ANTNTN $\mid$ AA-3' consensus. Furthermore, a distance of 15 bp most frequently separates the two nicks for L1, and between 13 to 16 bp for the different SINEs. The target site consensus of RTE1_LA$\mathrm{CAA}_{(\mathrm{n})}$ presents interesting similarities of primary sequence and structure with that of L1. First, like L1, the first nick occurs between a T and an $\mathrm{A}$, and the second nick is flanked on the $3^{\prime}$ by an A. Moreover, RTE1_LA-CAA $(\mathrm{n})$ also shows inter-nick distance preference (11 bp) and its insertion involves the recognition of two different sequence patterns around the first $\left(5^{\prime}-\operatorname{TTTN}_{(9)} \mathrm{T} \mid \mathrm{A}-3^{\prime}\right)$ and second $\left(5^{\prime}-\mid \mathrm{A}-3^{\prime}\right)$ nicking sites (Fig. 4f). These common features in target recognition probably reflect functional similarities between the AP endonuclease, and other potential factors involved in target recognition and cleavage, of L1 and RTE1_LA-CAA $(\mathrm{n})$. Our results, therefore, further underscore the conclusions of Ichiyanagi and Okada (2008) according to which vertebrate NLRs encoding AP endonuclease probably all share similar mechanisms for the initiation of TPRT.

\subsubsection{Similarities in target site consensus between RTE1_LA-CAA $A_{(n)}$ and AfroSINE(Anc)}

The target site consensuses of RTE1_LA-CAA $(\mathrm{n})$ and AfroSINE(Anc) are globally very similar. They both comprise three conserved Ts in the 5 ' flank that might correspond to a binding site, they both end with a conserved A in the immediate vicinity of the second nick, and both show a conserved distance of $21 \mathrm{bp}$ between the last conserved $5^{\prime} \mathrm{T}$ and the second nick (Fig. 4e and f). Notably, these features are not shared by other elements from the RTE family such as BovB (cow), RTE_Mdo (opposum) and Expander1_DR (zebrafish) (See Fig. 3 in Ichyanagi and Okada, 2008). We therefore believe that these common features in target site consensus shared by the two elements support the view that AfroSINE(Anc) were mobilized by RTE1_LA-CAA $(\mathrm{n})$ rather than by another distantly related family of NLR.

\subsubsection{Differences in target site consensus between RTE1_LA-CAA $(n)$ and AfroSINE(Anc)}

Despite being globally similar, the target site consensuses of RTE1_LA-CAA $(\mathrm{n})$ and AfroSINE(Anc) show some interesting differences, unlike L1, L1Tc and ingi elements that have the same target site consensus as their associated SINEs (Jurka, 1997; Szak et al., 2002; Bringaud et al., 2004; Gentles et al., 2005; Bringaud et al., 2006). First, the position of the first nicking site of the RTE1_LA consensus is $10 \mathrm{bp} \mathrm{3'}$ of the three first conserved Ts and is situated between a T and an A, whereas that of the AfroSINE(Anc) is 9 bp 3' of the three first conserved Ts and precedes two Ts (Fig. 4e and f). Second, a conserved A flanks the second nicking site on the $3^{\prime}$ side for RTE1_LA-CAA $(\mathrm{n})$, whereas it is on the $5^{\prime}$ side for the AfroSINE(Anc) (Fig. $4 \mathrm{e}$ and $\mathrm{f}$ ). Length, primary sequence, and tertiary mRNA structure could explain the differences observed between the target sites of the two elements. However, these potential factors also likely apply to L1 and its associated SINEs, but are not responsible for any difference in target site recognition between these paired elements. Therefore, we rather suggest that RTE1_LA$\mathrm{CAA}_{(\mathrm{n})}$ and AfroSINE(Anc) target site consensuses most likely differ because they are terminated by a different tandem repeat motif (see Section 3.1).

For some elements, the region corresponding to these $3^{\prime}$ terminal tandem repeats is believed to interact via base pairing, after first strand cleavage, with the partially denaturated target DNA (Chaboissier et al., 2000; Anzai et al., 2005). Consistent with this, in the case of RTE1_LA- 
$\mathrm{CAA}_{(\mathrm{n})}$, first target cleavage $\left(5^{\prime}-\mathrm{T} \mid \mathrm{A}-3^{\prime}\right)$ of the bottom strand (corresponding to $5^{\prime}-\mathrm{T} \mid \mathrm{A}-3^{\prime}$ of the top strand in Fig. 4f) produces a $3^{\prime}-\mathrm{OH}$ bearing T which can pair with the 3 ' tail of the RTE1_LA-CAA $(\mathrm{n})$
mRNA (composed of CAA repeats). In contrast, the target site T cannot pair with the GGTTT repeats terminating the AfroSINE(Anc) element. This would explain why few (here, 16 out of 99; Fig. 3) canonical first
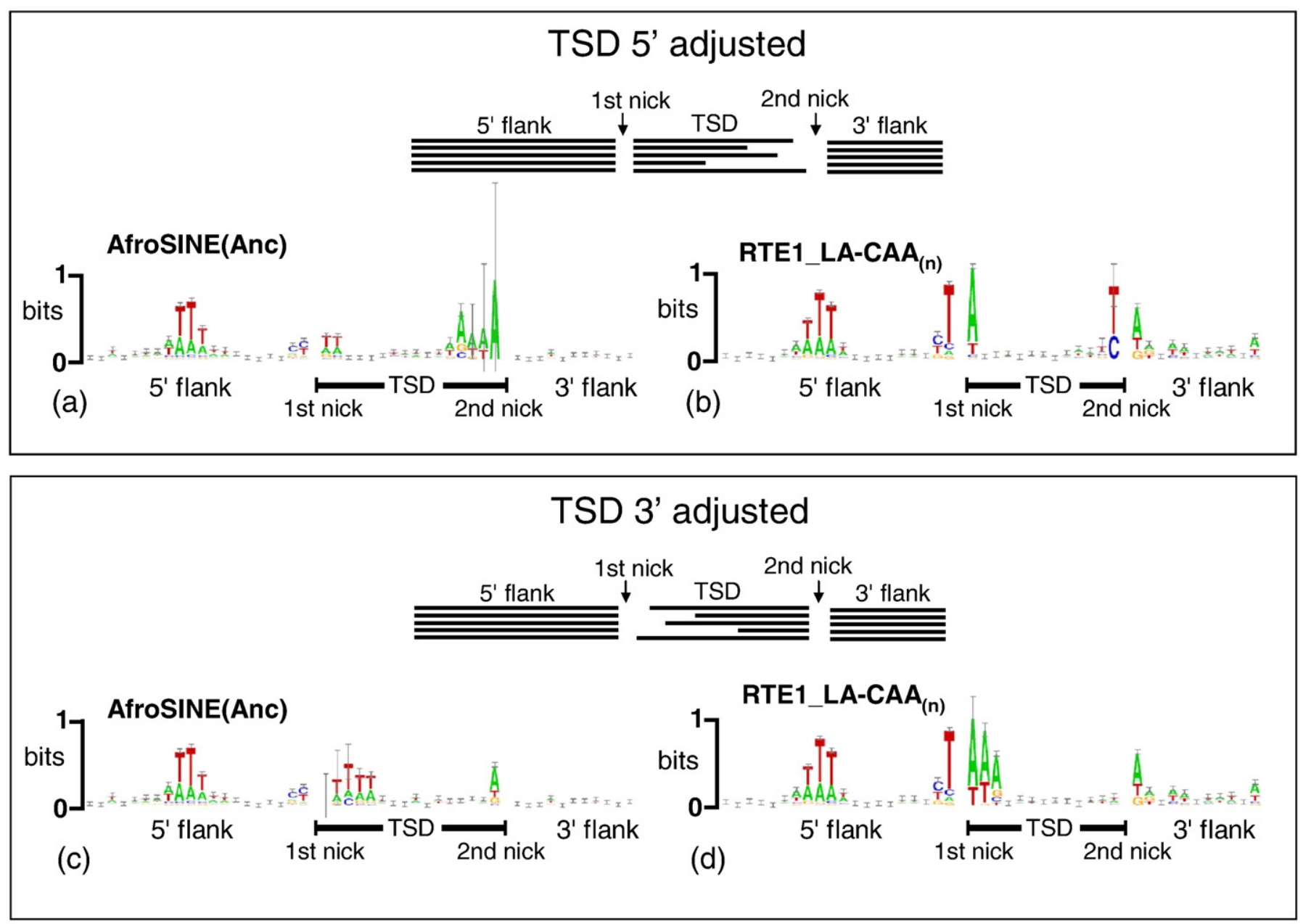

\section{Combined results}

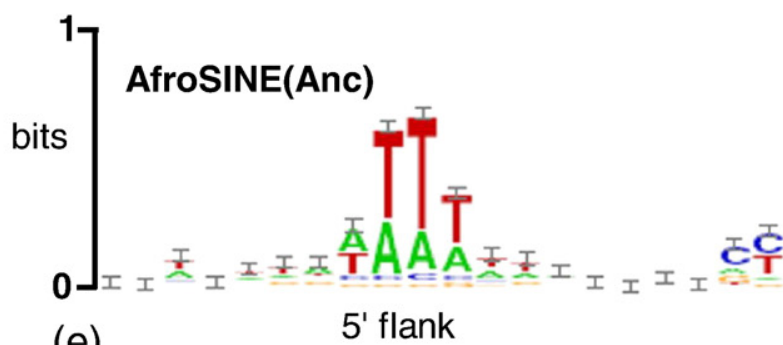

(e)

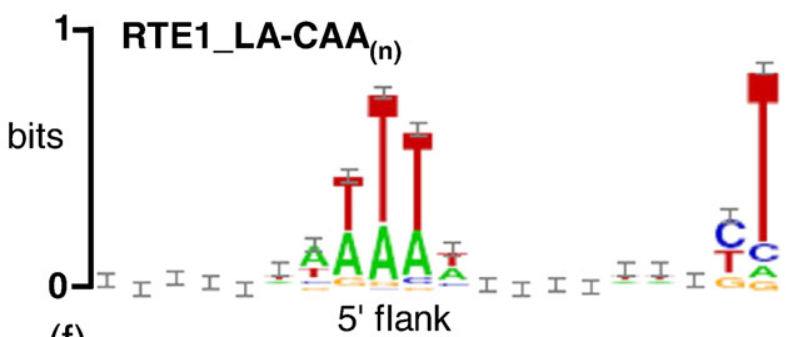

(f)

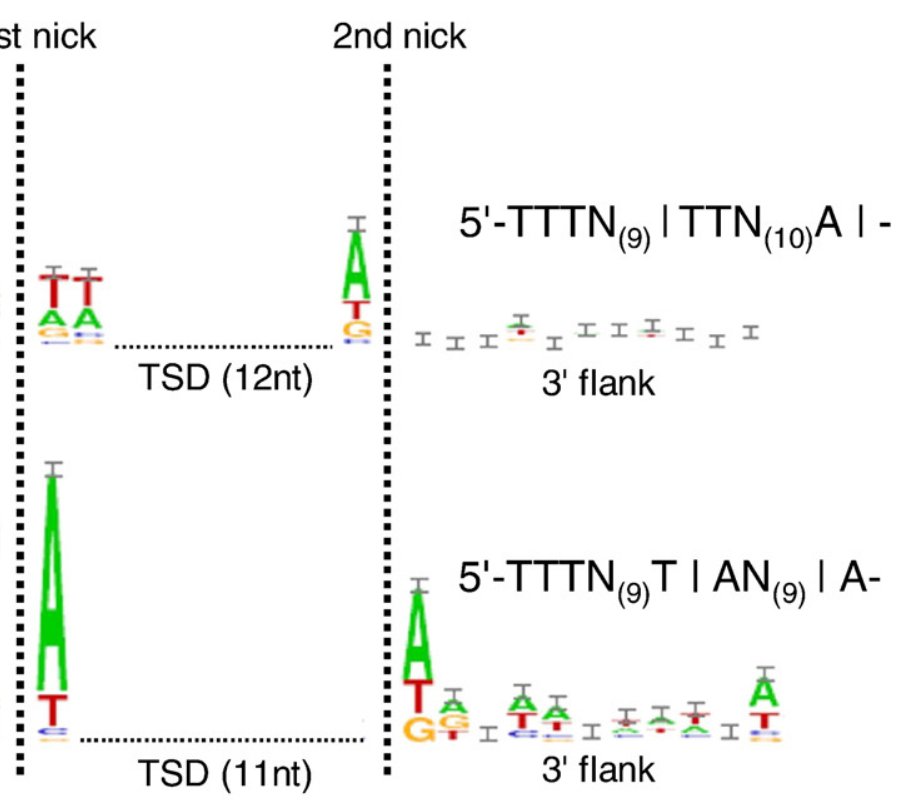


nickings of the RTE1_LA-CAA ${ }_{(n)}$ endonuclease (5'-T $\mid$ A-3' of the bottom strand) result in successful AfroSINE(Anc) insertions. The remaining 83 of 99 insertions do not match with the RTE1_LA consensus (5'-T |A-3', bottom strand). Notably, the vast majority of these had an apparent cut site that produced a target DNA suitable for pairing with the AfroSINE (Anc) GGTTT $_{(n)} 3^{\prime}$ tail, i.e. 5'-A $\left|\mathrm{N}-3^{\prime}, 5^{\prime}-\mathrm{AA}\right| \mathrm{N}-3^{\prime}$ or $5^{\prime}-\mathrm{CA} \mid \mathrm{N}-3^{\prime}$ (bottom strand). The observed differences in target site consensus between RTE1_LA-CAA $(\mathrm{n})$ and AfroSINE(Anc) can therefore most likely be explained by a high increase in integration success promoted by base pairing interaction between the $3^{\prime}$ terminal tandem repeats of the element mRNA and the target DNA, at the onset of TPRT (Chaboissier et al., 2000; Anzai et al., 2005). Consequently, these results confirm earlier suggestions (Jurka and Klonowski, 1996; Zingler et al., 2005; Repanas et al., 2007) and provide more evidence for a crucial role of the $3^{\prime}$ mRNA end in integration site selection.

\subsubsection{What role does the mRNA play in integration site choice?}

An interesting question is whether the element mRNA is a pre- or post-nicking determinant of integration site choice. In other words, does it actively influence the nicking specificity of the endonuclease prior to nicking via interaction with the target DNA and/or endonuclease and/or other co-factors to direct the endonuclease to produce an A-rich region that would facilitate base pairing with its GTrich 3' extremity? Or is it simply a passive, post-nicking constraint that would allow integration into only a subset of possible nicks, i.e. those that can be bound to the GT-rich 3' extremity?

As mentioned earlier, the preferred target site consensus for both RTE1_LA-CAA $(\mathrm{n})$ and AfroSINE(Anc) is rather loose, and shows a degree of nucleotide conservation that is similar to the target site consensuses of L1 and its associated SINEs (Jurka, 1997; Szak et al., 2002; Gentles et al., 2005). Consequently, the RTE1_LA-CAA $(\mathrm{n})$ endonuclease can cleave a large array of different sites. Moreover, although different, the RTE1_LA-CAA $(\mathrm{n})$ and AfroSINE(Anc) target site consensuses remain globally similar, and only very few changes are required to make the RTE1_LA-CAA $(\mathrm{n})$ consensus target site suitable for AfroSINE(Anc) insertion. The simplest modification to this effect would only involve a single base pair 3' "shift" of the first nick, which would produce a $\mathrm{A}^{\prime}{ }_{\mathrm{OH}}$ on the bottom strand of the target site. Instead of nicking at the most frequent site, $5^{\prime}-\mathrm{T} \mid \mathrm{A}-3^{\prime}$ on the bottom strand (see top strand Fig. 4f), the endonuclease would nick the target DNA just after the A of the bottom strand (before the T of the top strand). Other such nicks deviating from the most frequent one would simply involve one shift in the position of the first nick and one or two base misrecognitions. Our data do not allow us to discard a potential prenicking influence of the mRNA on the RTE1_LA-CAA $(\mathrm{n})$ endonuclease; but given that the EN can cleave at many sites others than its preferred one, and that only minor nicking errors are required to generate an AfroSINE(Anc) compatible insertion site, it seems more parsimonious to infer a post-nicking effect.

With this in mind, the difference in the position of the last $3^{\prime}$ conserved A relative to the second nicking site (see above and Fig. $4 \mathrm{e}$ and f) might simply be incidental to the slight deviation from the first most preferred nick made by the endonuclease. Supporting this view, Gentles et al. (2005) reported that the variations in nucleotide composition observed around the first and second nicking sites of L1 associated SINEs were correlated. Interestingly, as noted in Section
3.2.3, the last $3^{\prime}$ conserved $A$ is more frequently situated at a distance of $21 \mathrm{bp}$ from the third conserved $5^{\prime} \mathrm{T}$ for both elements (Fig. 4e and f). Thus although the presence of an A adjacent to the second nicking site seems somehow important, a distance factor, which would be more important than the exact position of this A, could explain the observed differences between the two consensus sequences.

\section{Conclusion}

This study shows that although AfroSINEs derive from RTE1_LA$\operatorname{GGTTT}_{(\mathrm{n})}$ elements, they were most likely mobilized by RTE1_LA$\mathrm{CAA}_{(\mathrm{n})}$. This provides us with the first example of a SINE/LINE pair for which the target site consensus is not identical between the two elements. Because of the apparent loose specificity of the endonuclease, the differences between the two are most probably due to the passive selection among a large spectrum of integration sites that occurs at the onset of the TPRT through post-nicking mRNA/target DNA interactions. According to this hypothesis, any SINE ending with a different tandem repeat motif than its LINE partner should also preferentially insert in a different target site. It will be interesting to verify this on several other SINE/LINE pairs ending with different types of tandem repeats, such as the UnaSINE2/UnaLINE2 pair of the eel (Kajikawa and Okada, 2002; Kajikawa et al., 2005).

\section{Acknowledgments}

We thank Terry Robinson for providing the facilities used to carry out this study and acknowledge financial support from the National Research Foundation to TR and CG. We thank Mélanie Debiais-Thibaud and Aurélie Hua-Van for their stimulating and helpful discussions during the preparation of this manuscript. We are also grateful to Shawn Christensen, Cédric Feschotte, Blaine Thompson, and two anonymous reviewers for their constructive comments that greatly improved earlier versions of the manuscript.

\section{Appendix A. Supplementary data}

Supplementary data associated with this article can be found, in the online version, at doi:10.1016/j.gene.2008.08.013.

\section{References}

Aksoy, S., Williams, S., Chang, S., Richards, F.F., 1990. SLACS retrotransposon from Trypanosoma brucei gambiense is similar to mammalian LINEs. Nucleic Acids Res. 18, 785-792.

Anzai, T., Osanai, M., Hamada, M., Fujiwara, H., 2005. Functional roles of 30-terminal structures of template RNA during in vivo retrotransposition of non-LTR retrotransposon, R1Bm. Nucleic Acids Res. 33, 1993-2002.

Arcot, S.S., Wang, Z., Weber, J.L., Deininger, P.L., Batzer, M.A., 1995. Alu repeats, a source for the genesis of primate microsatellites. Genomics 29, 136-144.

Bringaud, F., et al., 2004. The ingi and RIME non-LTR retrotransposons are not randomly distributed in the genome of Trypanosoma brucei. Mol. Biol. Evol. 21, 520-528.

Bringaud, F., et al., 2006. The Trypanosoma cruzi L1Tc and NARTc Non-LTR retrotransposons show relative site specificity for insertion. Mol. Biol. Evol. 23, 411-420.

Burke, W.D., Calalang, C.C., Eickbush, T.H., 1987. The site-specific ribosomal insertion element type II of Bombyx mori (R2Bm) contains the coding sequence for a reverse transcriptase-like enzyme. Mol. Cell Biol. 7, 2221-2230.

Burke, W.D., Müller, F., Eickbush, T.H., 1995. R4, a non-LTR retrotransposon specific to the large subunit rRNA genes of nematodes. Nucleic Acids Res. 23, 4628-4634

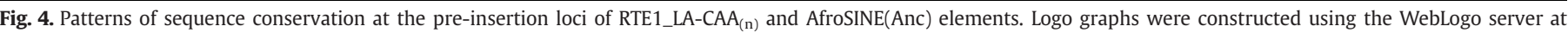

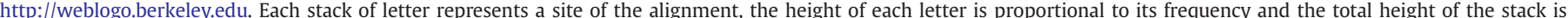

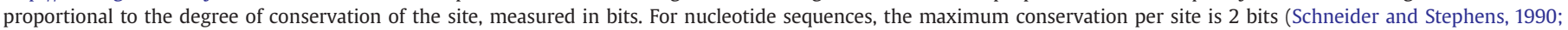

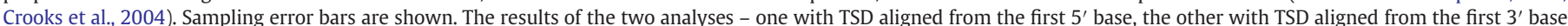

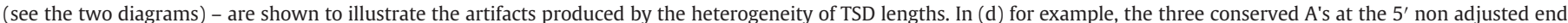

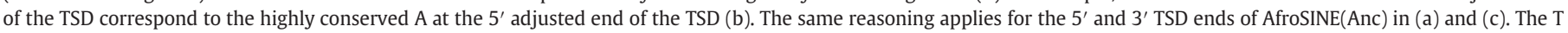

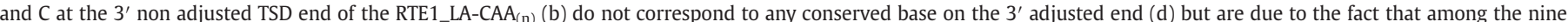

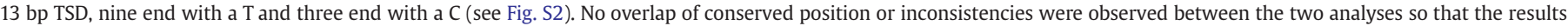

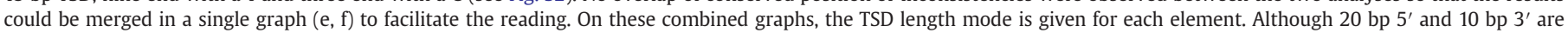
shown, $200 \mathrm{bp}$ of each were included in the analysis. Consensuses as written in the text are reported in the figure.
} 
Burke, W.D., Singh, D., Eickbush, T.H., 2003. R5 Retrotransposons insert into a family of infrequently transcribed 28S rRNA genes of planaria. Mol. Biol. Evol. 20, 1260-1270.

Chaboissier, M.C., Finnegan, D., Bucheton, A., 2000. Retrotransposition of the I factor, a non long terminal repeat of Drosophila, generates tandem repeats at the $3^{\prime}$ end. Nucleic Acids Res. 28, 2467-2472.

Christensen, S.M., Bibillo, A., Eickbush, T.M., 2005. Role of the Bombyx mori R2 element $\mathrm{N}$-terminal domain in the target-primed reverse transcription (TPRT) reaction. Nucleic Acids Res. 33, 6461-6468.

Cost, G.J., Boeke, J.D., 1998. Targeting of human retrotransposon integration is directed by the specificity of the L1 endonuclease for regions of unusual DNA structure. Biochemistry 37, 18081-18093.

Cost, G.J., Feng, Q., Jacquier, A., Boeke, J.D., 2002. Human L1 element target-primed reverse transcription in vitro. EMBO J. 21, 5899-5910.

Crooks, G.E., Hon, G., Chandonia, J.M., Brenner, S.E., 2004. WebLogo, a sequence logo generator. Genome Res. 14, 1188-1190.

Dewannieux, M., Esnault, C., Heidmann, T., 2003. LINE-mediated retrotransposition of marked Alu sequences. Nat. Gen. 35, 41-48.

Dewannieux, M., Heidmann, T., 2005. L1-mediated retrotransposition of murine B1 and B2 SINEs recapitulated in cultured cells. J. Mol. Biol. 349, 241-247.

Esnault, C., Maestre, J., Heidmann, T., 2000. Human LINE retrotransposons generate processed pseudogenes. Nat. Genet. 23, 363-367.

Feng, Q., Schumann, G., Boeke, J.D., 1998. Retrotransposon R1Bm endonuclease cleaves the target sequence. Proc. Natl. Acad. Sci. U. S. A. 95, 2083-2088.

Feschotte, C., Jiang, N., Wessler, S.R., 2002. Plant transposable elements: where genetics meets genomics. Nat. Rev. Genet. 3, 329-341.

Feschotte, C., Swamy, L., Wessler, S.R., 2003. Genome-wide analysis of mariner-like transposable elements in rice reveals complex relationships with stowaway miniature inverted repeat transposable elements (MITEs). Genetics 163, 747-758.

Gabriel, A., et al., 1990. A rapidly rearranging retrotransposon within the miniexon gene locus of Crithidia fasciculata. Mol. Cell Biol. 10, 15-624.

Garrett, J.E., Knutzon, D.S., Carroll, D., 1989. Composite transposable elements in the Xenopus laevis genome. Mol. Cell Biol. 9, 3018-3027.

Gentles, A.J., Kohany, O., Jurka, J., 2005. Evolutionary diversity and potential recombinogenic role of integration targets of non-LTR retrotransposons. Mol. Biol. Evol. 22, 1983-1991.

Gentles, A.J., et al., 2007. Evolutionary dynamics of transposable elements in the shorttailed opossum Monodelphis domestica. Genome Res. 17, 992-1004.

Gogolevsky, K.P., Vassetzky, N.S., Kramerov, D.A., 2008. Bov-B-mobilized SINEs in vertebrate genomes. Gene 407, 75-85.

Hall, T., 2004. Hall, T., 2004. BioEdit version 5.0.6. Available at, http://www.mbio.ncsu. edu/BioEdit/bioedit.html.

Higashiyama, T., Noutoshi, Y., Fujie, M., Yamada, T., 1997. Zepp, a LINE-like retrotransposon accumulated in the Chlorella telomeric region. EMBO J. 16, 3715-3723.

Ichiyanagi, K., Okada, N., 2008. Mobility pathways for vertebrate L1-, L2-, CR1-, and RTEclade retrotransposons. Mol. Biol. Evol. 25, 1148-1157.

Jurka, J., 1997. Sequence patterns indicate an enzymatic involvement in integration of mammalian retroposons. Proc. Natl. Acad. Sci. U. S. A. 94, 1872-1877.

Jurka, J., 2006. RTE1_LA, RTE-type sequence from African savanna elephant consensus. Repbase Reports 6, 164.

Jurka, J., Klonowski, P., 1996. Integration of retroposable elements in mammals, selection of target sites. J. Mol. Evol. 43, 685-689.

Kajikawa, M., Ichiyanagi, K., Tanaka, N., Okada, N., 2005. Isolation and characterization of active LINE and SINEs from the eel. Mol. Biol. Evol. 22, 673-682.

Kajikawa, M., Okada, N., 2002. LINEs mobilize SINEs in the eel through a shared $3^{\prime}$ sequence. Cell 111, 433-444.

Kazazian, H.H., 2004. Mobile elements, drivers of genome evolution. Science 303, 1626-1632.
Kojima, K.K., Fujiwara, H., 2003. Evolution of target specificity in R1 clade non-LTR retrotransposons. Mol. Biol. Evol. 20, 351-361.

Kurzynska-Kokorniak, A., Jamburuthugoda, V.K., Bibillo, A., Eickbush, T.H., 2007. DNAdirected DNA polymerase and strand displacement activity of the reverse transcriptase encoded by the R2 retrotransposon. J. Mol. Biol. 374, 322-333.

Laha, T., Brindley, P.J., Smout, M.J., Veritya, C.K., McManus, D.P., Loukas, A., 2002. Reverse transcriptase activity and untranslated region sharing of a new RTE-like, non-long terminal repeat retrotransposon from the human blood fluke, Schistosoma japonicum. Int. J. Parasitol. 32, 1163-1174.

Laha, T., Kewgrai, N., Loukas, A., Brindley, P.J., 2005. Characterization of SR3 reveals abundance of non-LTR retrotransposons of the RTE clade in the genome of the human blood fluke, Schistosoma mansoni. BMC Genomics 6, 154.

Lander, E.S., et al., 2001. Initial sequencing and analysis of the human genome. Nature 409, 860-921.

Luan, D.D., Eickbush, T.H., 1995. RNA template requirements for target DNA-primed reverse transcription by the R2 retrotransposable element. Mol. Cell Biol. 15, 3882-3891.

Luan, D.D., Korman, M.H., Jakubczak, J.L., Eickbush, T.H., 1993. Reverse transcription of R2Bm RNA is primed by a nick at the chromosomal target site, a mechanism for non-LTR retrotransposition. Cell 72, 595-605.

Malik, H.S., Eickbush, T.H., 1998. The RTE class of non-LTR retrotransposons is widely distributed in animals and is the origin of many SINEs. Mol. Biol. Evol. 15, 1123-1134.

Mandal, P.K., Bagchi, A., Bhattacharya, A., Bhattacharya, S., 2004. An Entamoeba histolytica LINE/SINE pair inserts at common target sites cleaved by the restriction enzyme-like LINE-encoded endonuclease. Eukaryotic Cell 3, 170-179.

Nikaido, M., Nishihara, H., Hukumoto, Y., Okada, N., 2005. Ancient SINEs from African endemic mammals. Mol. Biol. Evol. 20, 522-527.

Ohshima, K., Hamada, M., Terai, Y., Okada, N., 1996. The 3' ends of tRNA-derived short interspersed repetitive elements are derived from the $3^{\prime}$ ends of long interspersed repetitive elements. Mol. Cell Biol. 16, 3756-3764.

Ohshima, K., Okada, N., 2005. SINEs and LINEs, symbionts of eukaryotic genomes with a common tail. Cytogenet. Genome Res. 110, 475-490.

Repanas, K., Zingler, N., Layer, L.E., Schumann, G.G., Perrakis, A., Weichenrieder, O., 2007. Determinants for DNA target structure selectivity of the human LINE-1 retrotransposon endonuclease. Nucleic Acids Res. 35, 4914-4926.

Schneider, T.D., Stephens, R.M., 1990. Sequence logos, a new way to display consensus sequences. Nucleic Acids Res. 18, 6097-6100.

Smit, A.F., Riggs, A.D., 1995. MIRs are classic, tRNA-derived SINEs that amplified before the mammalian radiation. Nucleic Acids Res. 23, 98-102.

Szak, S.T., Pickeral, O.K., Makalowski, W., Boguski, M.S., Landsman, D., Boeke, J.D. 2002. Molecular archeology of L1 insertions in the human genome. Genome Biol. 3 (10).

Tu, Z., Li, S., Mao, C., 2004. The changing tails of a novel short interspersed element in Aedes aegypti, genomic evidence for slippage retrotransposition and the relationship between $3^{\prime}$ tandem repeats and the polydA tail. Genetics 168 2037-2047.

Volff, J.N., Körting, C., Froschauer, A., Sweeney, K., Schartl, M., 2001. Non-LTR retrotransposons encoding a restriction enzyme-like endonuclease in vertebrates. J. Mol. Evol. 52, 351-360.

Wei, W., et al., 2001. Human L1 retrotransposition: cis preference versus trans complementation. Mol. Cell Biol. 21, 1429-1439.

Zingler, N., Weichenrieder, O., Schumann, G.G., 2005. APE-type non-LTR retrotransposons, determinants involved in target site recognition. Cytogenet. Genome Res. 110, 250-268.

Zupunski, V., Gubensek, F., Kordis, D., 2001. Evolutionary dynamics and evolutionary history in the RTE clade of non-LTR retrotransposons. Mol. Biol. Evol. 18, 1849-1863. 